\title{
System Analysis and Reliability Analysis for Environmental Test Chambers
}

\author{
Chaoyang Gu, Lin Zhai, Wei Gu, Yuyu Sun, Chengjing Han, Xianwen Zhou, Wangqiang Niu \\ Received: November 28. Revised: December 29, 2020. Accepted: December 29, 2020.
}

\begin{abstract}
The purpose of this paper is to present the results of the application of various models to estimate the reliability of environmental test chambers, especially, the methodology proposed by the International Electrotechnical Commission (IEC), using the Homogeneous Poisson process (HPP) and Non-Homogeneous Poisson process (NHPP) models, is adopted first, and then, a non-monotonic trend test and bathtub curve intensity function not covered by IEC are used, and supplementary analysis is used to characterize the resulting failure intensity. For the first time, the stochastic process model was applied to evaluate the reliability of 20 environmental test chambers. The results show that the IEC standards process is suitable for the reliability evaluation of a single chamber, and 16 chambers conform to the HPP model and 4 chambers conform to the NHPP model. However, there is the power-law model (PLP) rejection cases among the overall description of multiple chambers by the IEC model. The rejected cases were analyzed again by using a non-monotonic trend test and constructing a double Weibull process tub curve strength function, and the 3-stage time interval of the bathtub curve failure is obtained, which is in line with the actual operation data. The Ward clustering method is used for the mean time between failures of 20 chambers, resulting in 4 types of chamber groups with different reliability values $(71,52,100,130$ days $)$, which is of great significance for studying the reliability of the environmental test chamber and carrying out customized maintenance.

Keywords-Systems Theory, Applied Systems Theory, Bathtub curve, Cluster analysis, Environmental test chamber , Non-homogeneous Poisson process, Reliability modeling.
\end{abstract}

\section{INTRODUCTION}

A $\mathrm{n}$ environmental test chamber is a complex repairable system, which is composed of multiple systems such as control, refrigeration, heating. Figure 1 is the physical diagram of an environmental test chamber, which is capable of high and low temperature damp heat test, with a nominal internal volume of $400 \mathrm{~L}$, a weight of $400 \mathrm{~kg}$, inner box size of W0.6m $\times$ $\mathrm{H} 0.85 \mathrm{~m} \times \mathrm{D} 0.8 \mathrm{~m}$, and an outer box size of $\mathrm{W} 1 \mathrm{~m} \times \mathrm{H} 1.7 \mathrm{~m} \times$ D1.4m. Environment test chambers are widely used in various industries[1], such as aerospace, information technique,

Chaoyang Gu is currently pursuing an M.S. degree with Shanghai Maritime University, Shanghai, China. His research direction is the reliability analysis of environmental test chambers.

Wangqiang Niu has been a Lecturer with Shanghai Maritime University, Shanghai, since 2008, he has been an Associate Professor, since 2017. His research interests include prognostics and health management (PHM), wireless power transfer, and control of marine equipment (ships and cranes). (corresponding author to provide e-mail: wqniu@shmtu.edu.cn). electronics, materials, electricity. Various performance indexes of different products or components are inspected under high temperatures, low temperatures, or damp-heat environments [2]. Taylor introduced a low-cost, $1.2 \mathrm{~m} \times 2 \mathrm{~m}$ forced ventilation microclimate test chamber to support the research and teaching of mechatronics [3]. Berchtold presented the selection method of the environmental test chamber to ensure the quality and reliability of the test chamber under various conditions of product testing [4].

The environmental test chamber is an important piece of equipment for product reliability testing, so the reliability of the test equipment itself puts forward a higher demand. The environmental test chamber must be kept in normal operation

Figure 1. The physical picture of an environmental test chamber

during the test period, or be overhauled and repaired timely

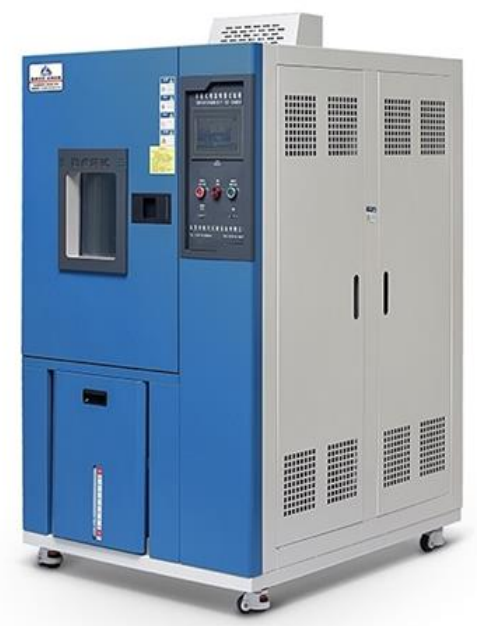

after an abnormality to avoid the impact on the test process and the accuracy of the results. Therefore, it is necessary to analyze and study the reliability of the environmental test chamber.

The environmental test chamber is an important piece of equipment for product reliability testing, so the reliability of the test equipment itself puts forward a higher demand. The environmental test chamber must be kept in normal operation during the test period, or be overhauled and repaired timely after an abnormality to avoid the impact on the test process and the accuracy of the results. Therefore, it is necessary to analyze and study the reliability of the environmental test chamber.

For the reliability modeling methods of repairable systems, a large number of statistical methods have been developed. 
Generally, the failure intensity $z(t)$ (Rate of occurrence of failures, ROCOF) of repairable systems can be estimated by the stochastic process and continuous failure interval time. The IEC standards give processing procedures and model selection process: the trend test method is used to test the trend of the data, if the test is no trend and the failure interval time is independent and identically distributed, $z(t)$ is a constant, and the homogeneous Poisson process model can be used for modeling. If there is a trend, the non-homogeneous Poisson process is used for modeling, and the power-law process model is used [5-7]. The statistical method of trend test provided by IEC standards is $U$ statistic verification. In the model of reliability analysis based on stochastic process, the IEC standards only reflect part of it. The model based on the Markov chain proposed by Kumamoto [8], the model based on Monte Carlo simulation proposed by Kurien et al. [9], Lewis proposed the branched Poisson process model studied [10], and the generalized updating process of "virtual age" introduced by Kijima [11]. Lee and Chang proposed a diagnosis method that can detect the presence and location of faults, and used machine learning algorithms based on time-frequency domain reflectometry to further distinguish faulty lines in multi-core cables [12]. A new online sequential extreme learning machine is based on the memory principle, which is essential to ensure the reliability and safety of aero engines introduced by $\mathrm{Lu}$ et al. [13]. Yan et al. discussed a time-varying reliability analysis method that combines the crossover method with the first-order reliability method [14]. Ren et.al proposed a method of uncertainty reliability evaluation combining GO-FLOW and dynamic Bayesian network (DBN) [15]. Mathematicians have provided a large number of models for reference [16-18]. Byrne and Lalanne justified the use of a solar photovoltaic air-conditioning ( $\mathrm{PV} \mathrm{AC)} \mathrm{system} \mathrm{coupled} \mathrm{to} \mathrm{a} \mathrm{latent} \mathrm{heat}$ thermal energy storage (LHTES)[19].

The non-homogeneous Poisson process adopted by IEC is a power-law model developed by Crow [20]. The expected number of failures in time $t$ is given by

$$
E[N(t)]=\lambda t^{\beta}
$$

where $\lambda$ is the scale parameter, $\beta$ is the shape parameter, the intensity function is

$$
z(t)=\frac{d}{d t} E[N(t)]=\lambda \beta t^{\beta-1}
$$

Given the reliability of the environmental test chamber, the independent international standard program should be adopted for reliability analysis. In this study, the maintenance record data of the environmental test chamber is analyzed by using various reliability models of the repairable system provided by IEC standards.

\section{CASE InTROdUCTION}

The environmental test chamber is a system composed of repairable parts. If a component fails, it can be repaired or replaced. Rigdon's research shows that for the maintenance of replacement parts, the system is reset to the initial state, that is, "repair as new" [21]. In this case, updating the process model and homogeneous Poisson process are the most suitable models.

20 environmental test chambers with the same technical design and structure were studied. The time of 2073 fault records is 7 years, 2013-2019. Since the chamber data are from different users, and some users have less than 5 times of maintenance, after eliminating the maintenance data with a small amount of data, the fault data reserved covers 20 environmental test chambers produced by the same company. Among them, 10076885-7 three test chambers have the same type and operating environment, and 02066502-4 have the same type and operating environment. The remaining 14 units belong to different companies, so the operating environment is different. The reliability of equipment with the different operating environment is analyzed by single fault maintenance data. Finally, 456 maintenance data were retained.

Fault location analysis is the first step in reliability analysis. By summarizing 456 maintenance data, failure modes are divided into 6 categories: electrical failure, humidification failure, refrigeration failure, control failure, waterway failure, and structural failure. The failure frequency of each part is shown in Figure 2a.

It can be seen from Figure 2a that the refrigeration system is the weak link of the 20 environmental test chambers. The refrigeration system is an important part of the environmental test chamber, and its stable performance will directly determine the accuracy of the environmental test. Through the statistical analysis, it is found that among 456 maintenance data, the refrigeration system and control system are high-risk systems.

To further understand the failure mechanism of the environmental test chamber, taking the refrigeration failure with a high incidence of faults as an example, a deeper failure cause analysis is carried out.

The failure frequency of the refrigeration system is shown in Figure $2 \mathrm{~b}$. It can be seen from the figure that the refrigerant $(23.3 \%)$, condenser $(31.6 \%)$, compressor $(9.1 \%)$ and solenoid valve $(13.3 \%)$ are the main fault causes, and the main fault causes account for $77.3 \%$ of the total fault causes. The condenser failure is the most common fault.

Through the statistical analysis of 20 environmental test chambers, it is found that the environmental test chambers have similar failure modes. After analyzing the maintenance data, the failure modes can be divided into 6 categories, and the refrigeration system is found to be the weak link of the environmental test chambers. In preventive maintenance, it can be considered to increase the number of spare parts of the refrigeration system to improve maintenance efficiency. 

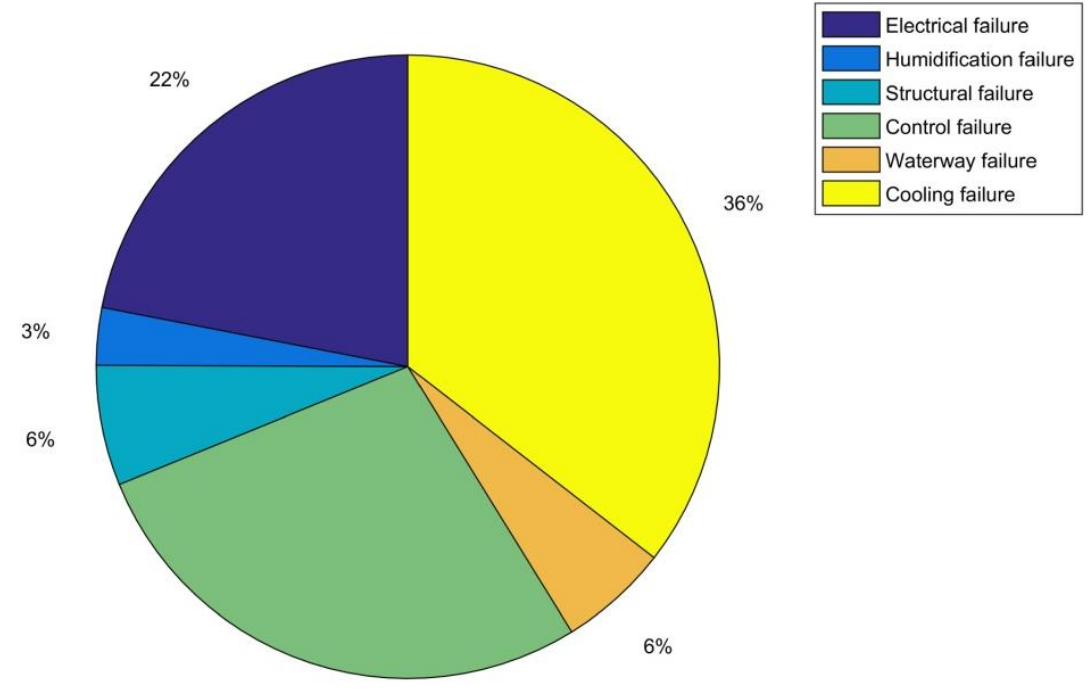

$28 \%$

(a)

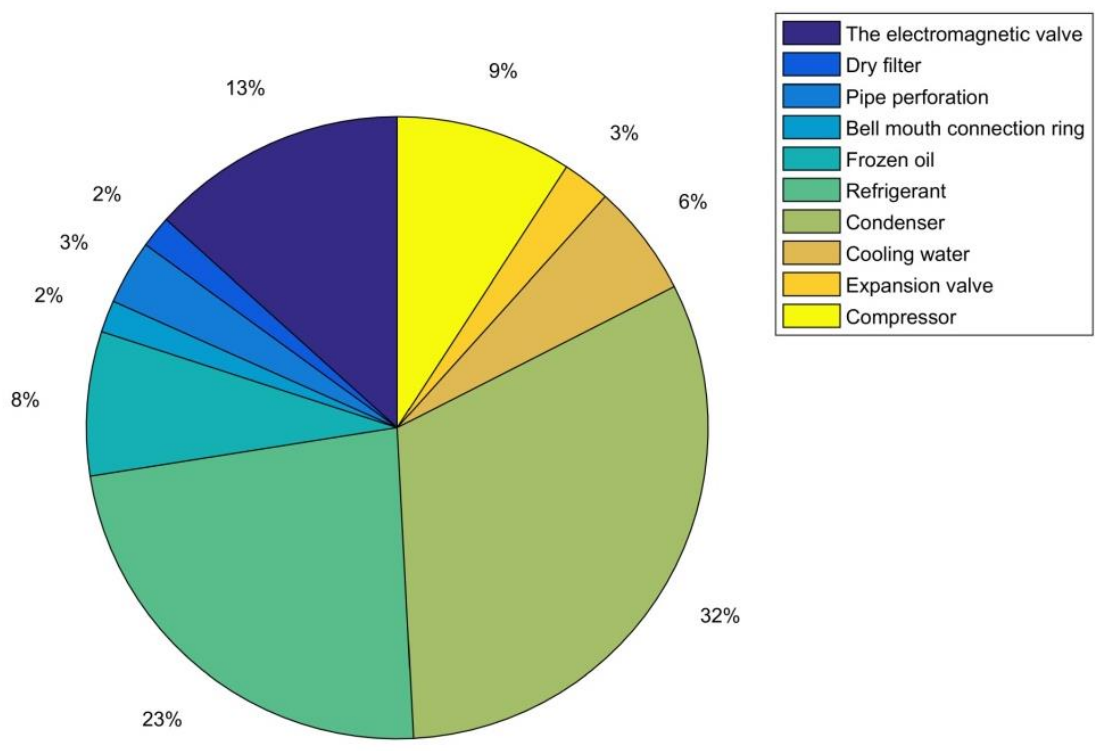

(b)

Figure 2. Failure modes and causes of environmental test chamber: (a) Environmental test chamber failure mode frequency diagram;(b) Environmental test chamber failure cause frequency diagram.

Through the above analysis, 456 maintenance data with the same failure mode are obtained, and the overall reliability of the environmental test chamber is analyzed. Since the environmental test chamber is worn with time, variable time is used to study the reliability. Table 1 shows the detailed data.

Since most studies assume the reliability of similar equipment is consistent in the same operating environment, it is generally considered that data deletion is an appropriate recommendation $[22,23]$. Navas et al. found that the results contradict this assumption for complex repairable systems [24]. This study also proves this viewpoint. Therefore, this study does not sample the limited population and does not eliminate the fault record data even if the initial data looks abnormal. 
INTERNATIONAL JOURNAL OF CIRCUITS, SYSTEMS AND SIGNAL PROCESSING

Table 1. Reliability Data Analysis and Summary of the Environmental Test Chamber

\begin{tabular}{ccc}
\hline $\begin{array}{c}\text { Equipment Number of the } \\
\text { test chamber }\end{array}$ & Total operation days & $\begin{array}{c}\text { Total } \\
\text { failures }\end{array}$ \\
\hline 10076885 & 883 & 16 \\
10076886 & 2259 & 40 \\
10076887 & 893 & 16 \\
02066502 & 724 & 6 \\
02066503 & 884 & 13 \\
02066504 & 905 & 20 \\
09056346 & 1046 & 14 \\
$12056351 \mathrm{~A}$ & 866 & 14 \\
03107322 & 1630 & 21 \\
09097144 & 812 & 8 \\
10076882 & 818 & 10 \\
02107323 & 883 & 12 \\
08076800 & 459 & 10 \\
03138198 & 1109 & 8 \\
04066519 & 785 & 8 \\
05056328 & 696 & 7 \\
12087046 & 805 & 12 \\
04087012 & 535 & 8 \\
09127982 & 522 & 9 \\
07097053 & 812 & 8 \\
\hline
\end{tabular}

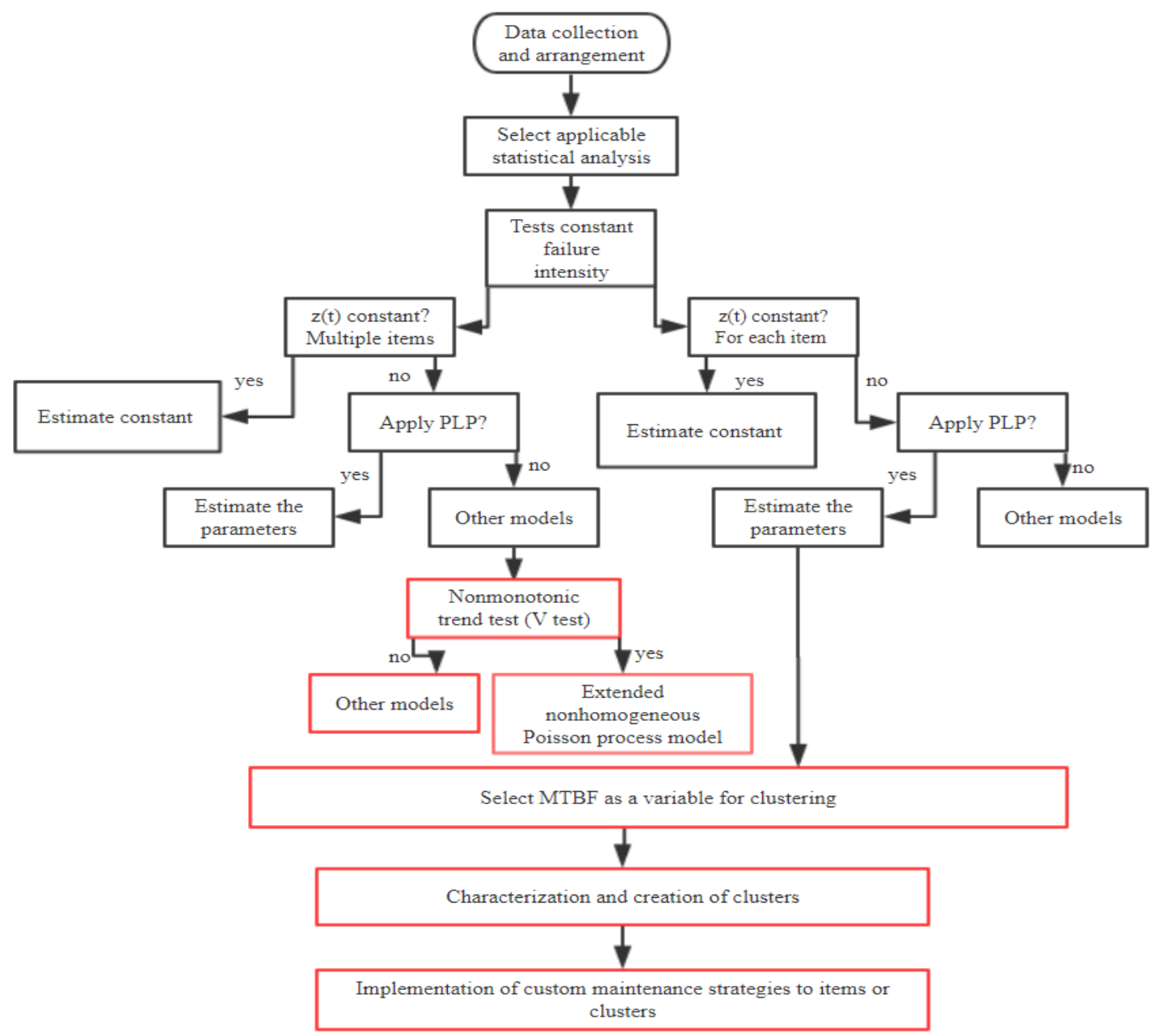

Figure 3. IEC standards and extended program for reliability modeling of Repairable System 
This study firstly applies IEC standards procedures to model the multiple chamber reliability of 2 groups of chambers of the same type and then modeling the reliability of a single chamber for 20 chambers. For the multiple test chamber data which does not pass the IEC standards, non-monotonic trend test, and non-homogeneous Poisson process model expansion are carried out. Finally, the cluster analysis of 20 test chambers is carried out to facilitate the customization of the maintenance strategy. The process is shown in Figure 3.

\section{RELIABILITY ANALYSIS OF ENVIRONMENTAL TEST CHAMBERS WITH THE METHODS OF THE IEC STANDARDS}

Firstly, according to IEC standards, the reliability model of 20 sets of test chambers with the same model and operating environment is established by using multiple test chamber parameter estimation methods. The failure behavior of all the same chamber is explained as a whole. If the model provided by IEC standards is rejected, it should try to analyze and process the single test chamber independently and model the reliability of each test chamber. Therefore, this section tests the model of IEC standards.

\section{A. Reliability Analysis of IEC Standard for Multiple Test Chamber}

The reliability analysis process provided by IEC standards points out that the overall reliability analysis of multiple test chambers should be carried out first. Therefore, this section carries out reliability analysis for test chambers of the same type and the same operating environment.

a. Trend Test of U Statistic of Failure Intensity Function for Multiple Test Chamber

According to IEC 60605-6, ed3.0 (2007) Section 7.3, multiple item $\mathrm{U}$ trend test procedure was carried out:

$$
U=\frac{\sum_{i=1}^{k} \sum_{j=1}^{r_{i}} T_{i j}-0.5\left(r_{1} T_{1}^{*}+r_{2} T_{2}^{*}+\ldots+r_{k} T_{k}^{*}\right)}{\sqrt{\frac{1}{12}\left(r_{1} T_{1}^{* 2}+r_{2} T_{2}^{* 2}+\ldots+r_{k} T_{k}^{* 2}\right)}}
$$

where $k$ is the number of equipment, $\mathrm{r}$ is the number of equipment failures, $T^{*}$ is the total operation time of each equipment, $T_{i j}$ is the $j$ th fault occurrence time of the $i$ th test chamber. The IEC standard states that if the absolute value of $U$ is greater than a given critical value, the assumption of constant failure intensity for all the same test chambers is rejected. Otherwise, the assumption will not be rejected.

The 3 test chambers of the $10076885-7$ series belong to the same company and have the same operation and operation environment. The calculation process is as follows:

$$
U=\frac{\sum_{i=1}^{3} \sum_{j=1}^{r_{3}} T_{i j}-0.5 *(16 * 883+40 * 2259+\ldots+893 * 16)}{\sqrt{\frac{1}{12}\left(16 * 883^{2}+40 * 2259^{2}+\ldots+16 * 893^{2}\right)}}
$$

Therefore, multiple test chamber failure intensity trend test is carried out for these 3 test chambers, and $U=4.53$ is obtained by the $U$ statistic test. Under the significant level of $\alpha=0.1$, the absolute value of test statistics exceeds the critical value of $U=$ 1.64, taking a larger positive value, Therefore, it is concluded that the assumption that the failure intensity is constant is rejected, and the failure trend increases.

0206502-4 series of 3 test chambers also belong to the same company of the same model, $U=2.05$ is obtained by $U$ statistics inspection publicity. Under the significance level of $\alpha=0.1$, the absolute value of test statistics exceeds the critical value of $U=$ 1.64, and a larger positive value is taken. Therefore, it is concluded that the assumption that the failure intensity is constant is rejected, and the failure trend increases.

It should be pointed out that the IEC standards have collected the Laplace test and graphic program $M(T)$ for the failure intensity trend test method, but not for a more robust trend test and non-monotonic trend test method.

b. Application of Multiple Test Chambers Power-Law Model

For the multiple test chamber data that passed the trend test, the model was applied. The $U$-statistics was used to test the trend of failure of 3 test chambers in each group. The results showed that the failure trend increased. The power-law model (PLP) is applied to the 2 groups of test chambers.

The shape parameter $\beta$ is estimated first, then the scale parameter $\lambda$ is found.

According to IEC standard 61710ed2.0 (2013) part 7.2.2 and 7.3.11, for multiple items, the following formula is used for iterative estimation of the shape parameter $\beta$ :

$$
\frac{N}{\beta}+\sum_{i=1}^{N} \ln t_{i}-\frac{N \sum_{j=1}^{k} T_{j}^{\beta} \ln T_{j}}{\sum_{j=1}^{k} T_{j}^{\beta}}=0
$$

where $t_{i}$ is the time of the $i$ th fault, $\mathrm{N}$ is the total number of faults accumulated in the test, $k$ is the total number of equipment, and $T_{j}$ is the equipment $\mathrm{j}(\mathrm{j}=1,2, \ldots, \mathrm{k})$.

The 3 test chambers of the same model, 10076885-7, get $\beta=1.3168$, which is greater than 1 , indicating an increasing trend. Another 3 test chambers of the same model, 02066502-4, find $\beta=1.5585$, which is greater than 1 , indicating also an increasing trend. The scale parameter $\lambda$ is obtained by the following formula:

$$
\lambda=\frac{N}{\sum_{j=1}^{k} T_{j}{ }^{\beta}}
$$

$10076885-7$ series 3 test chambers have $\lambda=0.001064$, and $02066502-4$ series 3 test chambers get $\lambda=0.0004408$.

According to the IEC standard PLP model.

10076885-7 series failure intensity function is:

$z(t)=0.001064 * 1.3168 t^{1.3168-1}$

$02066502-4$ series failure intensity function is:

$$
z(t)=0.000440822 * 1.5585 t^{1.5585-1}
$$

The fitness test is given in IEC 61710ed2.0 (2013) is Cramer-von Mises statistics, the formula is as follows:

$$
C^{2}=\frac{1}{12 M}+\sum_{j=1}^{M}\left[\left(\frac{t_{j}}{T}\right)^{\beta}-\left(\frac{2 j-1}{2 M}\right)\right]^{2}
$$

When it is timing truncated data, $M=N, T=T^{*}$. When it is 
censored data, $M=N-1, T=T_{n}$, where $M$ is the number of failures and $T$ is the total cumulative time. $t$ is the time of each failure.

Table 2. Multiple Test Chambers Trend Test and Model Acceptance

\begin{tabular}{cccccc}
\hline Equipment number & $\boldsymbol{U}$ & Model & No trend & $\boldsymbol{C}^{2}$ & $\begin{array}{c}\text { PLP Model } \\
\text { assumptions }\end{array}$ \\
\hline $10076885-7$ & 4.53 & PLP & reject & 0.697 & reject \\
$02066502-4$ & 2.05 & PLP & reject & 0.147 & accept \\
\hline
\end{tabular}

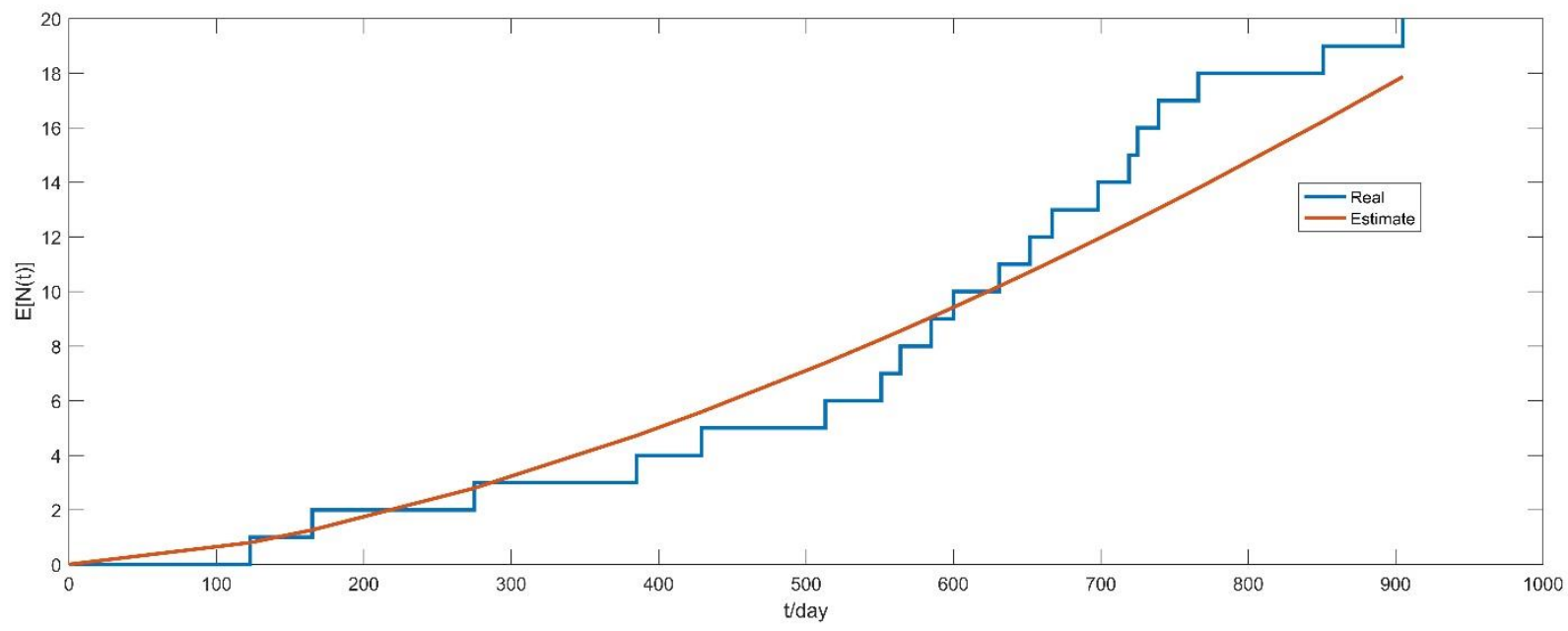

Figure 4. Actual value and the estimated value of failure times of 3 test chambers of $0206652-4$ series

The critical value of the significance level of $10 \%$ is selected. If the calculated value exceeds the critical value, then the assumption that the PLP model meets the test data must be rejected. The calculation process is as follows:

$$
C^{2}=\frac{1}{12 * 72}+\sum_{j=1}^{72}\left[\left(\frac{t_{j}}{2259}\right)^{1.3168}-\left(\frac{2 j-1}{2 * 72}\right)\right]^{2}
$$

The $10 \%$ significant level threshold is 0.1730 . It is calculated that 3 test chambers of the $10076885-7$ series $C^{2}=0.697$ are larger than the critical value. Therefore, the assumption that the PLP model meets the data is rejected. After analyzing the maintenance records and the failure data, it is found that the failure data of the 3 test chambers integrated by the total test time method may have a non-monotonic trend change. Therefore, a non-single-point trend test and an extended non-homogeneous Poisson process model are considered in Table 2.

The calculations for 3 test chambers of the $02066502-4$ series $C^{2}=0.1476$ are less than the critical value. Therefore, the assumption that the PLP model meets the data is accepted. According to the failure intensity function, the cumulative number of failures of the model is compared with the actual number of failures as shown in Figure 4 . It can be seen intuitively that the estimated value of the model is relatively close to the actual value.

By analyzing the original maintenance records, it can be seen that most of the maintenance activities of the 02066502-4 series of the 3 test chambers only involve some parts of the system, so the state after the repair of the system is close to the state before the repair, that is, "repair as old". The time between failures of the test chamber is no longer independent and identically distributed. It is found that the system state tends to deteriorate with the increase of time by the model solution value, which conforms to the actual test chamber operation process.

\section{B. Reliability Analysis of IEC Standards Process for a Single Test Chamber}

The IEC standards point out that when the reliability model of several test chambers is rejected, the reliability analysis of each test chamber should be considered. To accurately describe the reliability of each test chamber, the IEC standard process is used to analyze the reliability of 20 test chambers.

\section{a. Individual Test Chamber Trend Inspection}

This selected 20 environmental test chamber maintenance records. Because the test chamber of the same type has only 3 sets of the 2 groups processed above as well as the same operating condition, and there are rejected data for the model provided by the multiple test chambers IEC standards, the reliability of a single test chamber should be analyzed to understand fully the behavior of each chamber.

Perform trend verification on a single test chamber according to the following formula provided by the IEC standards:

$$
U=\frac{\sum_{i=1}^{r} T_{i}-r \frac{T^{*}}{2}}{T^{*} \sqrt{\frac{r}{12}}}
$$


where $r$ is the number of failures of each test chamber, $T^{*}$ is the total operating time of each test chamber, and $T_{i}$ is the time of each failure of the $i$ th test chamber. Choose the significance level $\alpha=0.1$, and the critical value of $U_{\alpha}$ is 1.64 . As a result, it is found that the failure intensity function values of 16 of the 20 test chambers showed no trend and did not exceed the critical value. Therefore, the IEC standards assume that the time between failures is exponentially distributed, and a

Table 3. $U$ Trend Test Results of a Single Chamber

\begin{tabular}{cccc}
\hline Equipment number & $\boldsymbol{U}$ & Model & No trend \\
\hline 10076885 & 1.4300 & HPP & Accept \\
10076886 & 4.4900 & NHPP & Reject \\
10076887 & 0.3107 & HPP & Accept \\
02066502 & 1.0782 & HPP & Accept \\
02066503 & 0.1921 & HPP & Accept \\
02066504 & 2.1338 & NHPP & Reject \\
09056346 & 1.1577 & HPP & Accept \\
12056351 A & 0.4650 & HPP & Accept \\
03107322 & 1.1035 & HPP & Reject \\
09097144 & 1.9230 & NHPP & Accept \\
10076882 & 1.2680 & HPP & Reject \\
02107323 & 0.4790 & HPP & Accept \\
08076800 & -0.8680 & HPP & Accept \\
03138198 & 1.7200 & NHPP & Reject \\
04066519 & -0.2043 & HPP & Accept \\
05056328 & 0.8051 & HPP & Accept \\
12087046 & 0.1670 & HPP & Accept \\
04087012 & -0.2541 & HPP & Accept \\
09127982 & 0.9026 & HPP & Accept \\
07097053 & 1.2971 & HPP & Accept \\
\hline & & &
\end{tabular}

The results show that the environmental test chamber is closer to "perfect repair", and the failure parts of the environmental test chamber are replaced during maintenance. However, the preventive maintenance of the environmental test chamber is very little, which leads to the failure intensity function close to a constant value.

\section{b. Estimation of the Constant for Each Test Chamber without} a Trend

According to the above trend test results, 16 test chambers do not show trends, so the HPP model is chosen, and the parameter estimation process is here.

According to the IEC standard, the constant value of the failure intensity function for 16 test chambers that do not show a trend is estimated according to the following formula:

$$
z(t)=\lambda=\frac{r}{T^{*}}
$$

homogeneous Poisson process model can be used to estimate the constant value $\lambda$. The assumption that the $U$ statistic test of the remaining 4 test chambers leads to a constant failure intensity is rejected and indicates an increase in failure intensity (as shown in Table 3), so the Non-homogeneous Poisson process model (HPP) is adopted.

where $r$ is the total number of failures, and $T^{*}$ is the total running time. The model assumes that the times between failures (TBF) has an exponential distribution, and the IEC standard does not include the necessary tests for the exponential distribution of time between failures. Therefore, the K-S test at the $5 \%$ significance level is used, and the $\lambda$ value and the K-S test results are shown in Table 4.

For 16 test chambers that did not show trends, the results passed the K-S test of the TBF exponential distribution hypothesis, so the hypothesis of TBF independent and identical distribution was met, and the fault maintenance data can be described by using the HPP model. Fig. 5 shows the comparison between the actual number of failures and the estimated number of failures for one of the chambers, which can directly prove the effectiveness of the model. The data are presented in 5 Table 
INTERNATIONAL JOURNAL OF CIRCUITS, SYSTEMS AND SIGNAL PROCESSING

Table 4. $\lambda$ Value of no Trend Test Chamber and K-S Test Result of TBF Exponential Distribution

\begin{tabular}{|c|c|c|c|c|}
\hline $\begin{array}{c}\text { Equipment } \\
\text { number }\end{array}$ & $\lambda$ & $\begin{array}{c}\text { D Critical } \\
\text { value }\end{array}$ & $\begin{array}{c}\text { D Observed } \\
\text { value }\end{array}$ & $\begin{array}{c}\text { Whether to } \\
\text { accept }\end{array}$ \\
\hline 10076885 & 0.01817 & 0.4313 & 0.1222 & Accept \\
\hline 10076887 & 0.01771 & 0.3050 & 0.1225 & Accept \\
\hline 02066502 & 0.00828 & 0.4981 & 0.1336 & Accept \\
\hline 02066503 & 0.01470 & 0.3521 & 0.0881 & Accept \\
\hline 09056346 & 0.01338 & 0.3261 & 0.1128 & Accept \\
\hline $12056351 \mathrm{~A}$ & 0.01580 & 0.3261 & 0.1651 & Accept \\
\hline 03107322 & 0.01280 & 0.2662 & 0.1749 & Accept \\
\hline 10076882 & 0.01220 & 0.3857 & 0.1632 & Accept \\
\hline 02107323 & 0.01360 & 0.3521 & 0.1215 & Accept \\
\hline 08076800 & 0.02170 & 0.3857 & 0.2019 & Accept \\
\hline 04066519 & 0.01020 & 0.4313 & 0.1721 & Accept \\
\hline 05056328 & 0.01005 & 0.4611 & 0.2302 & Accept \\
\hline 1208704 & 0.01490 & 0.3521 & 0.1169 & Accept \\
\hline 0408701 & 0.01495 & 0.4313 & 0.1444 & Accept \\
\hline 0912798 & 0.01724 & 0.4066 & 0.1415 & Accept \\
\hline 0709705 & 0.00985 & 0.4313 & 0.3375 & Accept \\
\hline
\end{tabular}

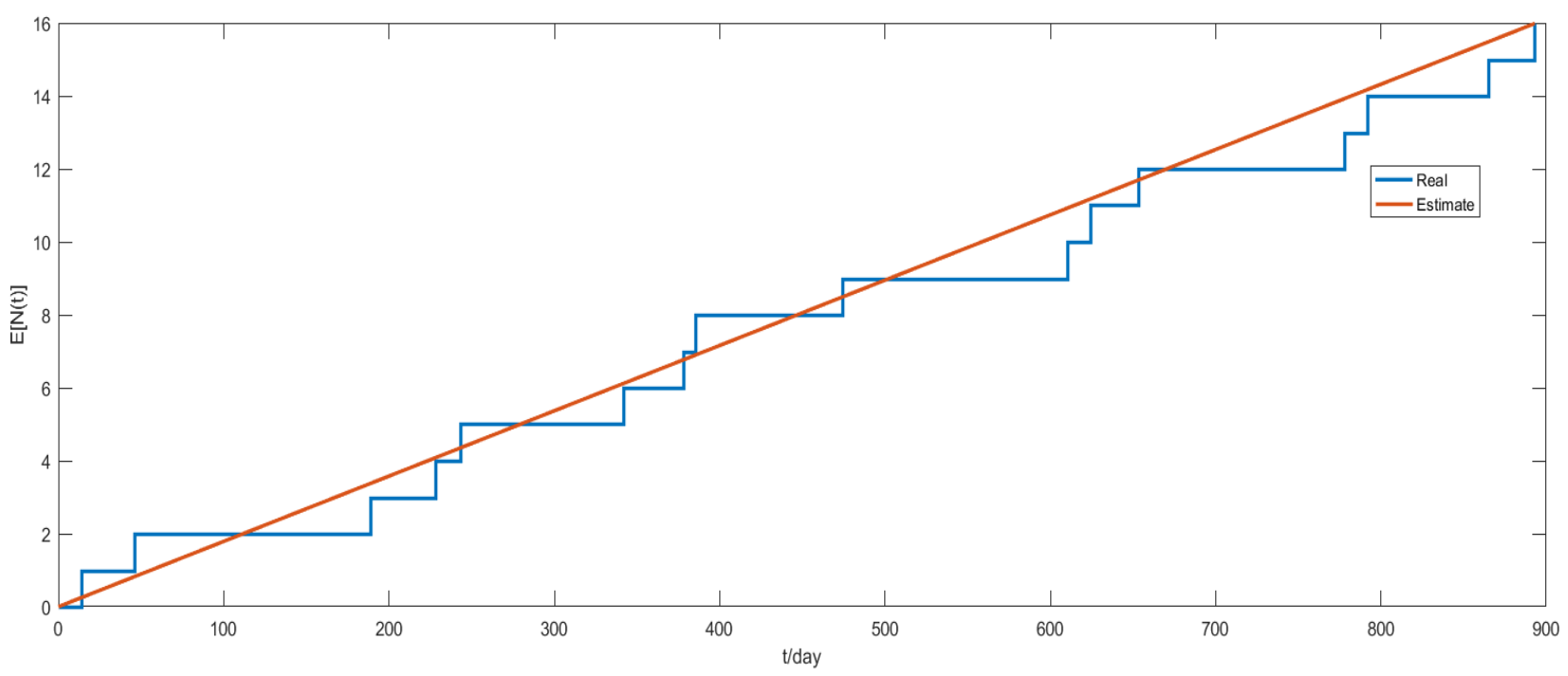

Figure 5. The actual value of the number of failures of a single non-trend test chamber and the estimated value of the model

Table 5. The Actual Value of the Number of Failures of a Single Non-trend Test Chamber and the Estimated Value of the Model

$\begin{array}{cccccc}\text { Real } & 2 & 4 & 6 & 8 & 10 \\ \text { Estimated } & 0.832 & 4.081 & 6.121 & 6.891 & 10.919\end{array}$


c. Application of PLP Model in a Single Trending Test Chamber

For the 20 chambers failure maintenance data analyzed, 4 of them passed the $U$ statistic trend test, indicating an increasing trend of failure. The 4 chambers' failure data were processed by using the PLP model.

According to IEC61710ed2.0 (2013). The values of $\beta$ were estimated respectively for the 4 chambers showing the trend of $U$ statistics of a single item. Calculated by the following formula:

$$
S_{1}=\sum_{j=1}^{N} \ln \left(\frac{T^{*}}{t_{j}}\right)
$$

Among them, $N$ is the number of failures, $T^{*}$ is the total running time, and $t_{j}$ is the $j$ th failure time. Calculate the unbiased estimate of $\beta$ and $\lambda$ according to the following formula:

$$
\beta=\frac{N-1}{S_{1}}, \lambda=\frac{N}{k\left(T^{*}\right)^{\beta}}
$$

Check the degree of fit for the solved parameters, and select the Cramer-von Mises statistics adopted by the IEC standards

The PLP model parameter values and $C^{2}$ test results of a single test chamber obtained from the above calculations are shown in Table 6. The results show that there is a trend that all the assumptions of the 4 test chambers PLP model are accepted. Fig. 6 shows the comparison between the actual number of failures of one of the test chambers and the estimated number of failures of the model. The data are presented in Table 7. It can be seen intuitively that the growth rate of the number of failures of the test chamber increases with time, which conforms to the description of the PLP model with increasing failure trends.

For repairable systems, the repaired environmental test chamber may be in 2 states: "repair as new" and "repair as old". The PLP model parameters $\beta$ obtained by the above 4 test chambers are all greater than 1 , indicating that the reliability has a decreasing trend, which is in line with the result of increasing failure trend indicated by the trend test. Observing the maintenance records of the above 4 test chambers discovered that the maintenance activities only involve some components, and repeated failures occur so that the failure interval no longer meets independent and identical distribution. Therefore, the PLP model can be used to describe the maintenance data.

By comparing the reliability results of a single test chamber with that of multiple test chambers, it is found that the 020660502-4 series chamber is PLP model in the reliability analysis of multiple test chambers, but 02066502 and 02066504 belong to the HPP model, and only 02066503 belongs to PLP model in the reliability analysis of single test chamber. Therefore, it is suggested that when the overall number of test chambers is small, the reliability analysis of each test chamber should be considered as far as possible.

Table 6. Parameter Values of Trending Test Chambers and the Results of Fitness Inspection

\begin{tabular}{cccccc}
\hline $\begin{array}{c}\text { Equipment } \\
\text { number }\end{array}$ & $\boldsymbol{\beta}$ & $\boldsymbol{\lambda}$ & $\boldsymbol{C}^{\mathbf{2}}$ & $\begin{array}{c}\boldsymbol{C}^{2} \text { Critical } \\
\text { value }\end{array}$ & $\begin{array}{l}\mathbf{1 0 \%} \\
\text { level } \\
\text { significance }\end{array}$ \\
\hline 10076886 & 3.3740 & $9.175 \mathrm{E}-11$ & 0.0584 & 0.172 & Accept \\
02066504 & 1.7256 & 0.000158 & 0.0805 & 0.172 & Accept \\
09097144 & 2.0376 & $9.425 \mathrm{E}-06$ & 0.0322 & 0.165 & Accept \\
03138198 & 1.8903 & $1.227 \mathrm{E}-05$ & 0.0615 & 0.165 & Accept \\
\hline
\end{tabular}

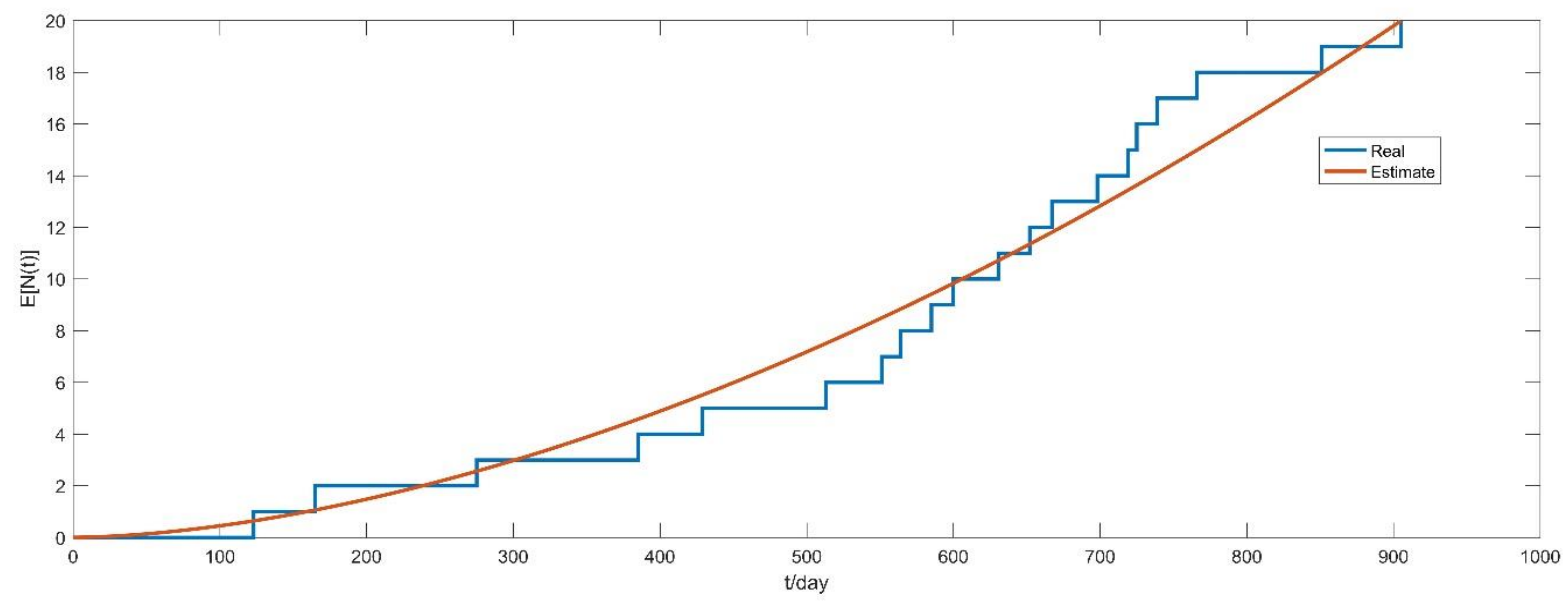


Figure 6. The actual value of the number of failures of a single trending test chamber and the estimated value of the model

Table 7. The Actual Value of the Number of Failures of a Single Trending Test Chamber and the Estimated Value of the Model

\begin{tabular}{cccccc}
\hline Real & 2 & 4 & 6 & 8 & 10 \\
Estimated & 1.059 & 4.572 & 7.503 & 8.836 & 9.831 \\
\hline
\end{tabular}

\section{Multiple Test Chambers Trend InSPeCtion AND MODEL EXPANSION (NON-IEC STANDARDS PROCESS)}

The above showed that within the existing environmental test chamber operating cycle data, the single test chamber fault maintenance data is suitable for the model proposed by the IEC standard, and the multiple test chamber power-law model provided by the IEC standard has data that fails the fit inspection. Navas et al. showed that a large number of repeated failures caused the rejection of the model provided by the IEC standards [24]. To facilitate the unified maintenance strategy management of a group of test chambers of the same model, it is necessary to expand the description of the multiple test chamber's reliability models.

\section{A. Non-monotonic Trend Test and Bathtub Curve Intensity Function Model}

Considering that the trend test method provided by the IEC standards is a monotonic trend test, subjectively, there may be non-monotonic changes in the test chamber failure intensity function trend. Ao et al. pointed out that in the actual trend test, the $U$ test, $V$ test, and $J$ test should all be used to comprehensively determine whether there is a trend [25]. Therefore, starting from the trend test, the supplementary analysis of the non-homogeneous Poisson process is carried out.

The 10076885-7 series 3 test chambers use the comprehensive trend test, and the $V$ test [26] is as follows:

H0: Homogeneous Poisson process.

H1: Non-monotonic trend.

$$
\begin{aligned}
& V_{1}=\frac{\sum_{i=1}^{n}\left|T_{i}-{ }^{*} / 2\right|-n T / 4}{T^{*} \sqrt{n / 48}} \\
& V_{2}=\frac{\sum_{i=1}^{n}\left|T_{i}-{ }^{*} / 2\right|-n T / 12}{{ }^{*}{ }^{2} \sqrt{n / 180}} \\
& V_{3}=2 \sum_{i=1}^{n} \ln \left(T /\left|2 T_{i}^{*}-T^{*}\right|\right)
\end{aligned}
$$

Substituting the data, it is found

$$
\begin{aligned}
& V_{1}=2.1786 ; V_{2}=1.7504 ; V_{3}=54.449 \\
& V_{1} \text { to } V_{3} \text { meets } \\
& V_{1}>0, V_{2}>0, V_{3}<2 n-0.66
\end{aligned}
$$

So, the failure data conforms to the bathtub curve of the non-monotonic trend.

For the bathtub curve failure process, according to Maurizio's research [27], this study assumes that the failure process consists of 2 Weibull processes. The failure intensity function is:

$$
z(t)=\frac{\beta_{1}}{\alpha_{1}}\left(\frac{t}{\alpha_{1}}\right)^{\beta_{1}-1}+\frac{\beta_{2}}{\alpha_{2}}\left(\frac{t}{\alpha_{2}}\right)^{\beta_{2}-1} \quad \alpha_{1}, \alpha_{2}, \beta_{1}, \beta_{2}>0
$$

Use the maximum likelihood estimation method to estimate the parameters of the intensity function of the $\mathrm{K}$ samples:

$$
L_{k}=\prod_{i=1}^{K}\left\{\prod_{j=1}^{n_{i}}\left\lfloor\frac{\beta_{1}}{\alpha_{1}}\left(\frac{t_{i j}}{\alpha_{1}}\right)^{\beta_{1}-1}+\frac{\beta_{2}}{\alpha_{2}}\left(\frac{t_{i j}}{\alpha_{2}}\right)^{\beta_{2}-1}\right\rfloor * \exp \left[-\left(\frac{T_{i}}{\alpha_{1}}\right)^{\beta_{1}}-\left(\frac{T_{i}}{\alpha_{2}}\right)^{\beta_{2}}\right]\right\}
$$

Therefore, an extended non-homogeneous Poisson process model is established, and the parameters of the 10076885-7 series failure intensity function are estimated:

$$
\begin{aligned}
& Z(t)=\left(\frac{t}{12.41}\right)^{0.67}+\left(\frac{t}{653.72}\right)^{1.89} \\
& z(t)=\frac{0.67}{12.41}\left(\frac{t}{12.41}\right)^{0.67-1}+\frac{1.89}{653.72}\left(\frac{t}{653.72}\right)^{1.89-1}
\end{aligned}
$$

The correlation coefficient between the fitted value of the bathtub curve model and the actual value is:

$$
r=\frac{\sum_{i=1}^{n}\left(t_{i}-\bar{t}\right) *\left(M_{i}-\bar{M}\right)}{\sqrt{\sum_{i=1}^{n}\left(t_{i}-\bar{t}\right)^{2} * \sum_{i=1}^{T}\left(M_{i}-\bar{M}\right)^{2}}}=0.986
$$

It can be seen from the value of the correlation coefficient that the model fit value is linearly related to the actual value, so it conforms to the assumption of the intensity function, that is, the bathtub curve model.

The most commonly used reliability index in production practice is the cumulative mean time between failures, which is defined as the mean time between failures in $(0, t]$ :

$$
M T B F=\frac{t}{Z(t)}
$$

In the above formula, MTBF is a single peak function. Assuming that the time at the peak is $t_{2}$, after $t_{2}$ the time between failures decreases, it can be considered that the reliability begins to decrease. This corresponds to the right turning point of the bathtub curve. The right turning point time $t_{2}$ is obtained by the following formula:

$$
(M T B F)^{\prime}=\frac{Z(t)-t^{*} z(t)}{Z^{2}(t)}=0
$$


According to the characteristics of the intensity function of the bathtub curve, it can be assumed that the slopes of the left and right turning points are opposite to each other [28]:

$$
z^{\prime}\left(t_{1}\right)=-z^{\prime}\left(t_{2}\right)
$$

Then $\mathrm{t} 1$ is the left turning point of the bathtub curve. From above, $t_{2}=2755.47$, the first derivative expression of the intensity curve is:

$$
\frac{d \omega(t)}{d t}=\frac{\beta_{1}\left(\beta_{1}-1\right)}{\alpha_{1}^{2}}\left(\frac{t}{\alpha_{1}}\right)^{\beta_{1}-2}+\frac{\beta_{2}\left(\beta_{2}-1\right)}{\alpha_{2}^{2}}\left(\frac{t}{\alpha_{2}}\right)^{\beta_{2}-2}
$$

The first derivative of the left turning point of the intensity function and the first derivative of the right turning point are opposite to each other, so $t_{1}=748.54$.
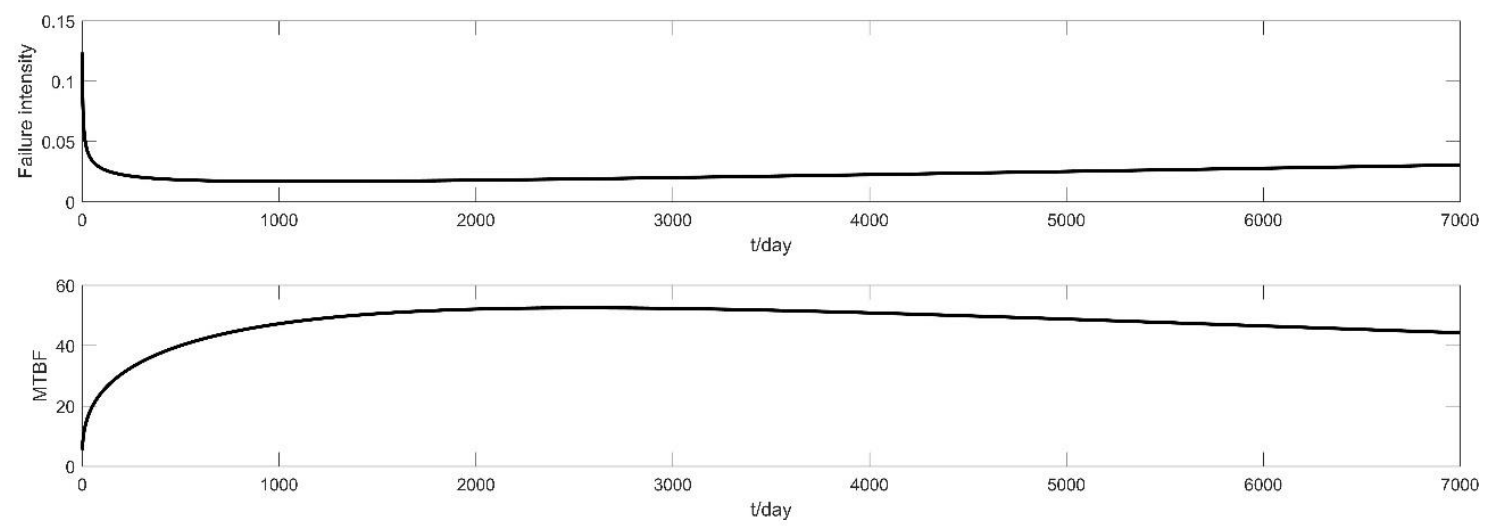

Figure 7. Intensity function curve and MTBF curve

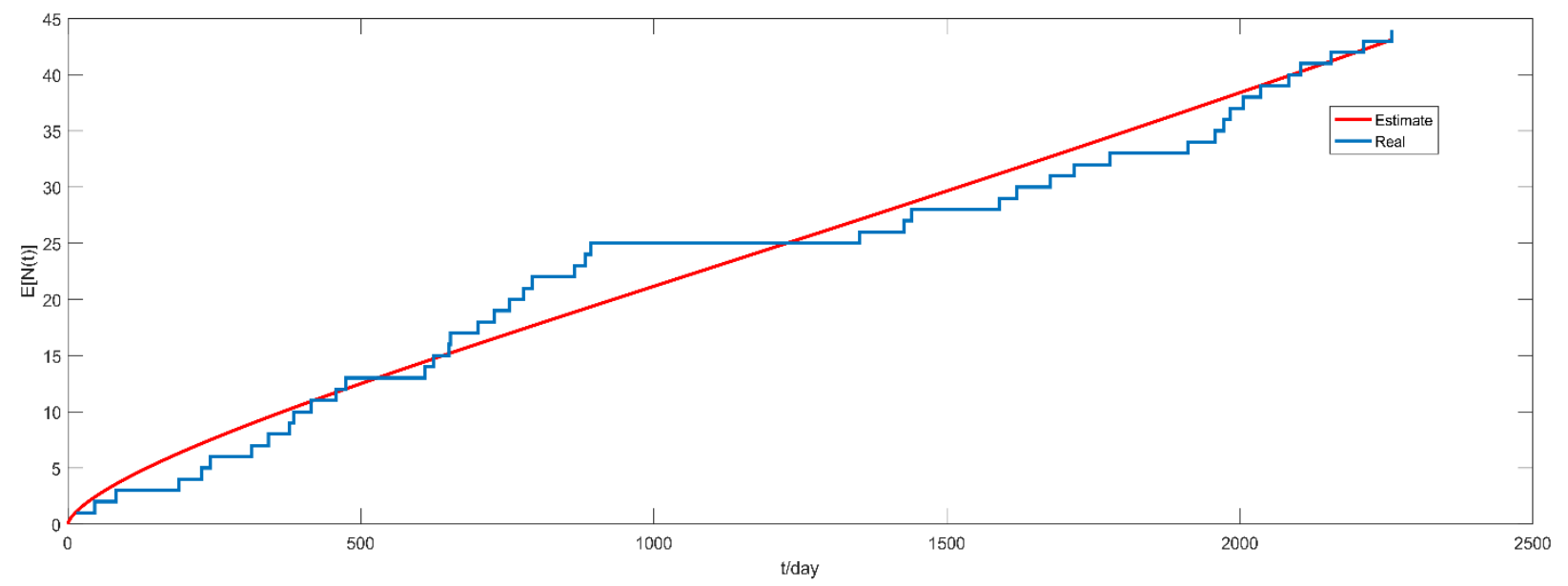

Figure 8. Actual and the estimated value of 10076885-7 series test chamber failure times

From the above calculation, it can be judged that the early failure period of this model of the environmental test chamber is about 2 years, and the cumulative MTBF (Mean time between failures) in the interval [748.54,2755.47] is solved to obtain the interval $[44.32,337.36]$ which is the change interval of the mean time between failures. It is consistent with the actual failure in statistical time.

It can be seen that before $t=748.54$, this type of environmental test chamber is in the early failure period from the mean time between failures (MTBF) curve (Fig. 7), the mean time between failures is small, the strength function is reduced, and the reliability is low. After 748.54 days, the change of intensity function is small, and the mean time between failures tends to be stable. After running for more than 2755.47 days, the mean time between failures decreases and the reliability reduces. At this time, it may enter the loss period.
Fig. 8 is the comparison between the estimated value and the actual value of the cumulative number of failures of 3 test chambers in the 10076885-7 series. It can be seen that the model conforms to the actual data trend. The extended non-homogeneous Poisson process model can be used to provide a holistic description of the 3 chambers' failure data.

From the analysis results of the single test chamber model in Section 3, it can be seen that the mean time between failures of the test chamber 10076885 is 54.36 days, the mean time between failures of 10076886 is 50.44 days, and the mean time between failures of 10076887 is 55.81 days. The overall mean time between failures of the 3 test chambers is 52.36 days, which is close to the mean time between failures of a single test chamber, so the overall description conforms to the actual reliability of these series. 


\section{B. Clusters of Repairable Environmental Test Chambers}

The data of this study comes from the maintenance company of environmental test equipment. For maintenance engineers, many test chambers need to perform reliability analysis. When performing reliability analysis, it is necessary to test the existing reliability model, carry out complex data analysis, and corresponding mathematical software support is lack. Therefore, the reliability cluster research of the test chambers with a similar mean time between failures can facilitate greatly the management of reliability test results and improve maintenance efficiency.

To simplify the maintenance management decision when the number of test chambers is large, it is first necessary to select a variable to establish clustering. To establish a cluster of environmental test chambers, this study selected the calculated mean time between failures as a variable and takes 20 test chambers as the research object. There are many existing clustering methods, and the Ward method has been proved to produce almost nothing to do with the distance metric used when creating clusters, so the Ward method is used for cluster creation [24].

The Ward clustering method is based on the idea of the sum of squares of deviation, which was proposed by Ward in 1936 and developed by Orloci in 1967 [29]. This method contains $N$ samples. The sum of deviation squares is as follows:

$\mathrm{S}=\sum_{i=1}^{n}\left(x_{i}-\bar{x}\right)^{*}\left(x_{i}-\bar{x}\right)$

then take $N$ samples as $N$ clusters, where $\mathrm{S}=0$, and merge two of them into one cluster, that is, one cluster at a time. The sum of squares of deviation increases with each reduction of one cluster, and the two clusters with the smallest increase in $S$ are chosen each time to merge until all samples are grouped.

Euclidean distance is used to cluster the interval time between the failures of 20 environmental test chambers. When the number of clusters is $2,3,4$, and 5 , the clustering results are as Table 8 .

The clustering pedigree chart intuitively shows the clustering process. Combined with the clustering spectrum and the distribution frequency of each class, 2 kinds of choices can be generated, 2 or 4 categories. To more accurately reflect the reliability of different groups, the clustering result is selected as 4 categories

When 20 environmental test chambers are clustered with the mean time between failures as variables and four clusters are selected as clustering results, 11, 9, 12, 7, 8, 18, 17, 5 is the first category, and the mean time between failures is 71.45 days. $13,6,19,2,3,1$ is the second type, and the mean time between failures is 52.79 days. 14,4 are the third type, and the mean time between failures is 129.73 days. $16,15,20,10$ is the fourth type, and the mean time between failures is 100.17 days.

8 test chambers $(1,2,3,4,5,6,10,11)$ belong to company A, which has the same operating environment but belong to different models. The models of test chamber 1, 2, and 3 are the same, which are classified into the second category. The models of test chambers 4,5 , and 6 are the same and are divided into 3 different categories. 4 test chambers $(17,18,19,20)$ belong to company $\mathrm{B}$ and have the same operating environment. Test chambers 17 and 18 are in the first category, chamber 19 belongs to the second category, while chamber 20 is in the fourth category.

The general assumption is that the expected reliability of the same system in the same operating environment should be very similar a priori for all systems under test. Through cluster analysis, it is found that the same equipment of the same company is divided into different groups, and the result conflicts with the hypothesis. The study by Weckman et al. [30] also found this.

For maintenance engineers, maintenance strategies can be grouped according to the clustering results. For groups with a mean time between failures of 100.17and129.73 days, relatively higher reliability, the period of preventive maintenance can be appropriately extended. For the 52.79-day and 71.45 days mean time between failures grouping, relatively lower reliability, additional preventive maintenance measures can be appropriately taken.

Table 8. Clustering Results

\begin{tabular}{|c|c|c|c|c|c|c|c|}
\hline \multicolumn{2}{|c|}{2} & \multicolumn{2}{|c|}{3} & \multicolumn{2}{|c|}{4} & \multicolumn{2}{|c|}{5} \\
\hline Count & Percent & Count & Percent & Count & Percent & Count & Percent \\
\hline 6 & $30.00 \%$ & 6 & $30.00 \%$ & 4 & $20.00 \%$ & 4 & $20.00 \%$ \\
\hline \multirow[t]{4}{*}{14} & $70.00 \%$ & 8 & $40.00 \%$ & 2 & $10.00 \%$ & 4 & $20.00 \%$ \\
\hline & & 6 & $30.00 \%$ & 6 & $30.00 \%$ & 4 & $20.00 \%$ \\
\hline & & & & 8 & $40.00 \%$ & 2 & $10.00 \%$ \\
\hline & & & & & & 6 & $30.00 \%$ \\
\hline
\end{tabular}




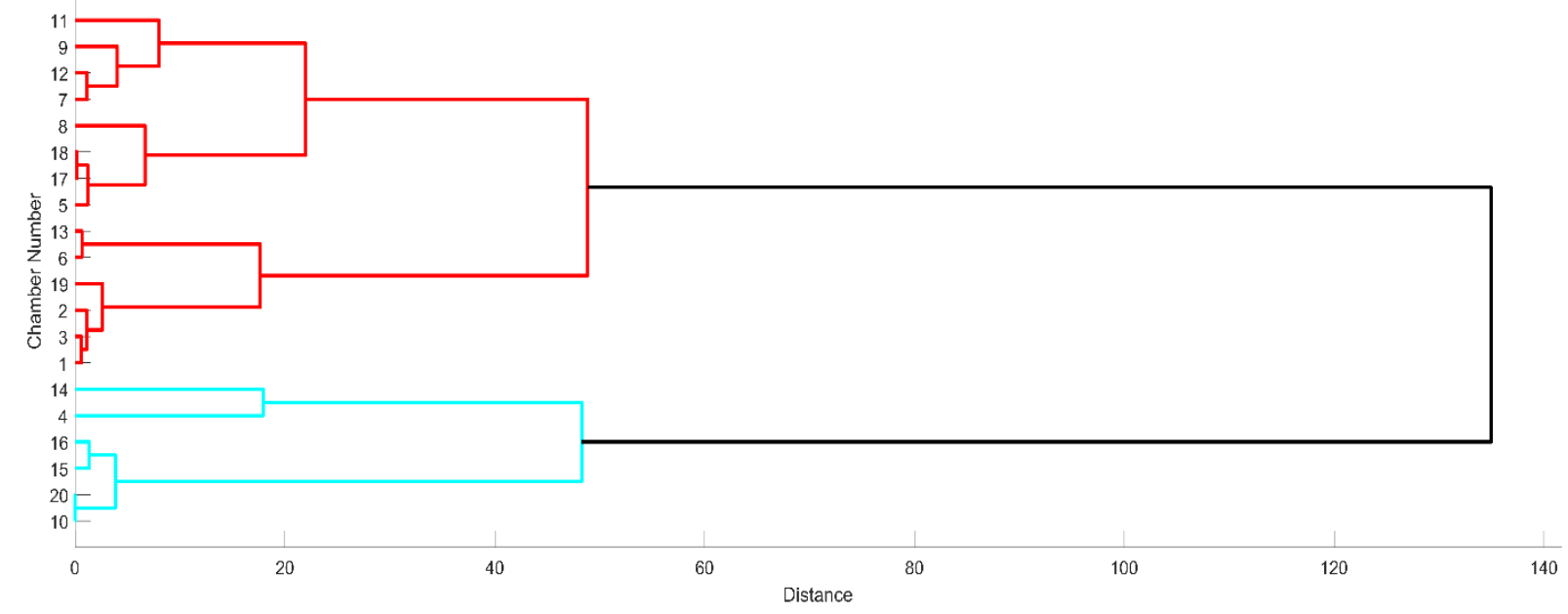

Figure 9. Ward clustering pedigree

\section{V.CONCLUSION}

Through the statistical analysis of fault maintenance data and taking expert advice, 456 fault maintenance data are selected. The failure modes of the environmental test chamber are divided into six categories, and it is found that the refrigeration system is the weak link of the environmental test chamber.

The IEC standard process is applied to the reliability analysis of the environmental test chamber for the first time. It is found that the standard process provided by IEC can be used for the reliability analysis of a single test chamber. If the multi-device PLP model is rejected, the non-monotonic trend of the data can be retested. If the results are nonmonotonic, the extended non-homogeneous Poisson process model, such as the failure strength function of the bathtub curve, can be used to describe the whole test chamber. The ward clustering method is used to select the mean time between failures (MTBF) as a variable to cluster and generate classes with close reliability, which provides the basis for a customized maintenance strategy.

This paper describes the statistical process of fault maintenance data of environmental test chambers, analyzes it by Poisson process, and obtains the reliability model based on random point process. The model can evaluate the failure time of the environmental test chamber, but it can not analyze the specific failure position and cause. Therefore, the subsequent reliability model might be established by using the Bayesian network. By analyzing the component level fault data, the importance degree of different components can be obtained, which is convenient for spare parts management, and accurate fault location.

\section{REFERENCES}

[1] J. Bojkovski, I. Pusnik, J. Drnovsek, and D. Hudoklin, "Automated system for evaluation of climatic chambers," IEEE Transactions on Instrumentation and Measurement, vol. 50, no. 6, pp. 1599-1603, 2002.

[2] J Xiaohai, Z Tiewei, and W Lijun, "Fault analysis and troubleshooting of French Klemme chamber based on PC control," Refrigeration and Air Conditioning: Sichuan, vol. 22, no. 005, pp. 80-81, 2008.

[3] C. J. Taylor, "Environmental test chamber for the support of learning and teaching in intelligent control," International Journal of Electrical Engineering Education, vol. 41, no. 4, pp. 375-387, Oct 2004.

[4] E. Berchtold, "Selecting an environmental test chamber," EE, Evaluation Engineering, vol. 43, no. 10, pp. 20-25, 2004.

[5] IEC 60300 ed2.0 (2011), "Dependability management", International Electrotechnical Commission (IEC), Geneva,2011

[6] IEC 60605 ed2.0 (2007), "Equipment reliability testing", International Electrotechnical Commission (IEC), Geneva, 2007

[7] IEC 61710ed2.0 (2013), "Power-law model - goodness-of-fit tests and estimation methods", International Electrotechnical Commission (IEC), Geneva, 2013

[8] H. Kumamoto, K. Tanaka, K. Inoue, and E. J. Henley, "State-Transition Monte Carlo for Evaluating Large, Repairable Systems," IEEE Transactions on Reliability, vol. R-29, no. 5, pp. 376-380, 2009.

[9] K. C. Kurien, G. S. Sekhon, and O. P. Chawla, "Analysis of Aircraft Reliability Using Monte Carlo Simulation," International Journal of Quality \& Reliability Management, vol. 10, no. 2, 1993.

[10] P. A. W. Lewis, "A branching Poisson process model for the analysis of computer failure patterns," J. Royal Statist. Soc., B, vol. 26, no. 3, pp. 398-456, 1964.

[11] M. Kijima, "Some results for repairable systems with general repair," Journal of Applied probability, pp. 89-102, 1989.

[12] C. K. Lee and S. J. Chang, "Fault Detection in Multi-Core C\&I Cable via Machine Learning-Based Time-Frequency Domain Reflectometry," Applied sciences, vol. 10, no. 1, p. 158, 2019.

[13] L. Junjie, H. Jinquan, and L. Feng, "Sensor Fault Diagnosis for Aero Engine Based on Online Sequential Extreme Learning Machine with Memory Principle," Energies, vol. 10, no. 1, pp. 39, 2017.

[14] M. Yan, B. Sun, B. Liao, Y. Ren, and M. Wei, "FORM and Out-Crossing Combined Time-Variant Reliability Analysis Method for Ship Structures," IEEE Access, vol. 6, no. 99, pp. 9723-9732, 2017.

[15] Y. Ren, D. Fan, X. Ma, Z. Wang, Q. Feng, and D. Yang, "A GO-FLOW and Dynamic Bayesian Network combination approach for reliability evaluation with uncertainty: A case study on a Nuclear Power Plant," IEEE Access, vol. 6, pp. 7177-7189, 2017. 
[16] S. T. Jiang, T. L. Landers, and T. R. Rhoads, "Semi-parametric proportional intensity models robustness for right-censored recurrent failure data," Reliability Engineering \& System Safety, vol. 90, no. 1, pp. 91-98, 2005.

[17] M. Kaminskiy and V. Krivtsov, "G1-renewal process as a repairable system model," Reliability: Theory \& Applications, vol. 5, no. 3 (18), pp.1-6, 2015.

[18] E. A. Peña, "Statistical Methods for the Reliability of Repairable Systems. (Book Reviews)," Journal of the American Statistical Association, vol. 97, no. 458, pp. 652-654, 2002.

[19] P. Byrne and P. Lalanne, "Simulation of a latent heat thermal energy storage for the solar air-conditioning system of a net-zero energy hotel," WSEAS Transactions on Environment and Development, vol. 15, pp. 510-526, 2019.

[20] L. H. Crow, "Reliability analysis for complex, repairable systems," ARMY MATERIEL SYSTEMS ANALYSIS ACTIVITYABERDEEN PROVING GROUND MD1975.

[21] S. E. Rigdon and A. P. Basu, Statistical Methods for the Reliability of Repairable Systems. Journal of the American Statistical Association, 97(458), pp. 652-653, 2000.

[22] M. Luo, M.-1. Wu, and X.-y. Wang, "Study on reliability test for brake control execution unit of the rail transit vehicle," in 2010 International Conference on E-Product E-Service and E-Entertainment, pp. 1-4: IEEE 2010.

[23] S. Chen, T. Ho, and B. Mao, "Reliability evaluations of railway power supplies by fault-tree analysis," IET Electric Power Applications, vol. 1, no. 2, pp. 161-172, 2007.

[24] M. A. Navas, C. Sancho, J. Carpio, and C. Madu, "Reliability analysis in railway repairable systems," International Journal of Quality \& Reliability Management, pp. 10-15, 2017.

[25] A. Changlin, L. Yijun, and Yan, "Reliability of tractor engine based on non-homogeneous Poisson process," Chinese Journal of Mechanical Engineering, no. 10, pp. 206-210, 2007.

[26] J. K. Vaurio, "Identification of process and distribution characteristics by testing monotonic and non-monotonic trends in failure intensities and hazard rates," Reliability Engineering \& System Safety, vol. 64, no. 3, pp. 345-357, 1999.

[27] G. Pulcini and M. Guida, "Reliability Analysis of Mechanical Systems With Bounded and Bathtub Shaped Intensity Function," IEEE Transactions on Reliability, vol. 58, no. 3, pp. 432-443, 2009.

[28] Z. Genbao, Z. Kunneng, and W. Yang, "Research on the modeling technology of the strength function bathtub curve of multiple CNC machine tools," Mechanical Science and Technology, vol.35, no.1, pp.104-108, 2016.

[29] C. Juarez, A. R. Messina, R. Castellanos, and G. Espinosa-Perez, "Characterization of Multimachine System Behavior Using a Hierarchical Trajectory Cluster Analysis," IEEE Transactions on Power Systems, vol. 26, no. 3, pp. 972-981, 2011.

[30] G. R. Weckman, R. L. Shell, and J. H. Marvel, "Modeling the reliability of repairable systems in the aviation industry," Computers \& Industrial Engineering, vol. 40, no. 1-2, pp. 51-63, 2001.

Chaoyang Gu received the B.E. degrees from Zhoukou Normal University, Zhoukou, China, in 2016. He is currently pursuing an M.S. degree with Shanghai Maritime University, Shanghai, China. His research direction is the reliability analysis of environmental test chambers.

Lin Zhai, is currently an associate professor at Shanghai Maritime University, Shanghai. Her main research direction is port equipment and ship automation and mechatronics technology.

Wei Gu received the B.E. and Ph.D. degrees from Shanghai Maritime University, Shanghai, China, in 1982 and 2008, respectively.

Since 1982, he has been a Teacher with Shanghai Maritime University, where he has been a Professor, since 1997, and is currently the Director of the Key Laboratory of Transport Industry of Marine Technology and Control Engineering. His research interest includes marine information control technology.

Yuyu Sun is a senior engineer at Jiangsu Automation Research Institut China, He engaged in research work in laboratory equipment management.

Chengjing Han is the chief engineer of Shanghai Sizhong Experimental Equipment Co., Ltd., Shanghai, China. He is engaged in the commissioning, maintenance, and repair of environmental test chambers with 16 years of experience.

Xianwen Zhou is an engineer of the Key Laboratory of Transport Industry of Marine Technology and Control Engineering, Shanghai Maritime University, Shanghai, China. He mainly engaged in reliability test research, product testing, ship and port automation design research, and reliability test equipment maintenance.

Wangqiang Niu received the B.E. degree from Xi'an Aerotechnical College, Xi'an, China, in 1998, the M.E. degree from Northwestern Polytechnical University, Xi'an, in 2004, and the Ph.D. degree from Shanghai Jiao Tong University, Shanghai, China, in 2008.

From 2013 to 2014, he was a Visiting Lecturer with McMaster University, Hamilton, ON, Canada. Since 2008, he has been a Lecturer with Shanghai Maritime University, Shanghai, where

\section{Creative Commons Attribution License 4.0 (Attribution 4.0 International, CC BY 4.0)}

This article is published under the terms of the Creative Commons Attribution License 4.0 https://creativecommons.org/licenses/by/4.0/deed.en_US 